TRANSACTIONS OF THE

AMERICAN MATHEMATICAL SOCIETY

Volume 354, Number 5, Pages 2115-2129

S 0002-9947(02)02941-0

Article electronically published on January 7, 2002

\title{
CERTAIN IMPRIMITIVE REFLECTION GROUPS AND THEIR GENERIC VERSIONS
}

\author{
JIAN-YI SHI
}

Dedicated to Professor Cao Xi-hua on his 80th birthday

\begin{abstract}
The present paper is concerned with the connection between the imprimitive reflection groups $G(m, m, n), m \in \mathbb{N}$, and the affine Weyl group $\widetilde{A}_{n-1}$. We show that $\widetilde{A}_{n-1}$ is a generic version of the groups $G(m, m, n)$, $m \in \mathbb{N}$. We introduce some new presentations of these groups which are shown to have some group-theoretic advantages. Then we define the Hecke algebras of these groups and of their braid versions, each in two ways according to two presentations. Finally we give a new description for the affine root system $\bar{\Phi}$ of $\widetilde{A}_{n-1}$ such that the action of $\widetilde{A}_{n-1}$ on $\bar{\Phi}$ is compatible with that of $G(m, m, n)$ on its root system in some sense.
\end{abstract}

Reflection groups have well-known classical presentations (see [2], 3], [5]). This gives a neat way to describe the reflection groups. But there might be some limitations. Here are two examples. One is for the imprimitive reflection groups $G(m, m, n), m, n \in \mathbb{N}$ (the set of positive integers) defined in 1.2. With respect to the presentation of $G(m, m, n)$ given in [2] (called the standard presentation of $G(m, m, n)$, see Fig.1), there is no known formula for its length function (see [1]). The other is for the affine Weyl groups $\widetilde{A}_{n-1}, n>1$, which form an infinite family of reflection groups (here and later we denote by $\widetilde{A}_{n-1}$ the affine Weyl group of type $\widetilde{A}_{n-1}$, by abuse of notation). With respect to the presentation of $\widetilde{A}_{n-1}$ given in [5, 4.7] (called the standard presentation of $\widetilde{A}_{n-1}$, see Fig.3), there is no natural way to regard $\widetilde{A}_{h}$ as a subgroup of $\widetilde{A}_{k}$ for $h<k$, comparing with the case of symmetric groups. Then it is natural to ask if there exist some other presentations of $G(m, m, n)$ and $\widetilde{A}_{n-1}$, again by generators and relations, such that the above obstructions could be overcome. In the present paper, we are concerned with the connection between the groups $G(m, m, n), m \in \mathbb{N}$, and their generic version $G(\infty, \infty, n)$ (which is isomorphic to $\widetilde{A}_{n-1}$ ). We give a new presentation for each of the groups $G(m, m, n)$ and $G(\infty, \infty, n)$. Then we use them to study these groups, the associated braid groups, Hecke algebras and root systems. As a consequence, an affirmative answer is given to the above question.

A generic version $G(\infty, \infty, n)$ for the groups $G(m, m, n), m \in \mathbb{N}$, is a certain monomial matrix group over the set $\left\{x^{k} \mid k \in \mathbb{Z}\right\}$ ( $x$ an indeterminate) such that

Received by the editors November 9, 1999 and, in revised form, May 24, 2001.

2000 Mathematics Subject Classification. Primary 20F55.

Supported partly by University of Notre Dame, and partly by the National Science Foundation of China, the Science Foundation of the University Doctorial Program of CNEC and the City Foundation for the Selected Academic Research of Shanghai. 
any $G(m, m, n)$ is its homomorphic image under the specialization $x=e^{2 \pi i / m}$ (see 1.5). $G(\infty, \infty, n)$ is isomorphic to a subgroup in the inverse limit of the inverse system formed by the groups $G(m, m, n), m \in \mathbb{N}$ (see 1.6). $G(\infty, \infty, n)$ can be regarded as the matrix form of $\widetilde{A}_{n-1}$ (see Theorem 2.3) and is "the Weyl group" of some algebraic group over the ring $\mathbf{k}\left[x, x^{-1}\right]$, where $\mathbf{k}$ is an algebraically closed field of positive characteristic (see [6] and 2.6). This form of $\widetilde{A}_{n-1}$ is useful in the study of its conjugacy problem (see [9, Sect. 3]).

For each of the groups $G(\infty, \infty, n)$ and $G(m, m, n)$, we introduce a new presentation by generators and relations (see Proposition 3.3, and $3.4(2)$ ). The new presentation of $G(\infty, \infty, n)$ (resp. $G(m, m, n))$ is compatible with the standard one of $G(m, m, n)$ (resp. $G(\infty, \infty, n))$ under the specialization map $\phi_{m}^{n}: G(\infty, \infty, n) \longrightarrow$ $G(m, m, n)$. The new presentation of $G(\infty, \infty, n)$ has the advantage that a lower rank member in the family is naturally a subgroup of any higher rank member. Also, the new presentation of $G(m, m, n)$ has the advantage that the length function can be formulated explicitly (see 3.5 ).

We define the Hecke algebras associated to the groups $G(\infty, \infty, n), G(m, m, n)$ and the associated braid groups, each in two ways according to two presentations (standard and new), which are shown to be isomorphic (see 4.1, 4.2 and Proposition 4.3). Moreover, the Hecke algebra of $G(m, m, n)(\operatorname{resp} . B(m, m, n))$ can be naturally regarded as a quotient of the Hecke algebra $\mathcal{H}(G(\infty, \infty, n)$ ) (resp. $\mathcal{H}(B(\infty, \infty, n))$ ) (see 4.4). Note that our definition for the Hecke algebra of $G(m, m, n)$ is slightly different from that of [2, the former involves one parameter and the latter involves two.

We describe the root system $\bar{\Phi}$ of $G(\infty, \infty, n) . \bar{\Phi}$ is essentially the affine root system of type $\widetilde{A}_{n-1}$ on which the group $G(\infty, \infty, n)$ acts naturally. It has the additional property that the action of $G(\infty, \infty, n)$ on $\bar{\Phi}$ is compatible with that of $G(m, m, n)$ on its root system $\Phi(m)$ via a pre-root system $\Phi$ and the specialization $x=e^{2 \pi i / m}($ see 5.2 and 5.5$)$.

The connection between the groups $G(\infty, \infty, n)$ and $G(m, m, n)$ makes it possible to generalize some results which were originally shown only for one of the groups $G(\infty, \infty, n)$ and $G(m, m, n)$, but now could be shared by both. For example, such results can be found easily in the representation theory, since any representation of $G(m, m, n)$ could be lifted to $G(\infty, \infty, n)$.

An analogous discussion can proceed on the imprimitive groups $G(m, 1, n), m \geqslant$ 1 , and their generic version $G(\infty, 1, n)$. We shall deal with it in a forthcoming paper.

The content of the paper is organized as follows. In Section 1, we introduce the generic version $G(\infty, 1, n)$ (resp. $G(\infty, \infty, n)$ ) for the imprimitive reflection groups $G(m, 1, n)$ (resp. $G(m, m, n))$. Then we concentrate ourselves only on $G(\infty, \infty, n)$ and $G(m, m, n)$ in the subsequent sections. We show the isomorphism $G(\infty, \infty, n) \cong \widetilde{A}_{n-1}$ in Section 2. For each of $G(\infty, \infty, n)$ and $G(m, m, n)$, we introduce a new presentation by generators and relations in Section 3 , and two associated Hecke algebras in Section 4; the latter are shown to be isomorphic. Finally, we give a new description for the affine root system of $G(\infty, \infty, n)$ in Section 5 .

\section{The GENERIC VERSIONS OF CERTAIN IMPRIMITIVE REFLECTION GROUPS}

Fix $n \in \mathbb{N}$. In the present section, we introduce the generic versions for two families of imprimitive reflection groups acting on $\mathbb{C}^{n}$. 
1.1. Let $V$ be an $n$-dimensional complex vector space with a unitary inner product ( $\mid$ ). A reflection in $V$ is by definition a unitary transformation $\sigma$ of $V$ of finite order with $\operatorname{dim}(1-\sigma) V=1$. A group $G$ generated by reflections in $V$ is called a reflection group. A reflection group $G$ is called Coxeter, if there exists a $G$-invariant $\mathbb{R}$-subspace $V_{0}$ of $V$ such that the canonical map $\mathbb{C} \otimes_{\mathbb{R}} V_{0} \longrightarrow V$ is bijective. A reflection group $G$ in $V$ is imprimitive, if there exists a decomposition $V=$ $V_{1} \oplus \cdots \oplus V_{r}$ of nontrivial proper subspaces $V_{i}, 1 \leqslant i \leqslant r$, of $V$ such that $G$ permutes $\left\{V_{i} \mid 1 \leqslant i \leqslant r\right\}$ (see [3]).

1.2. Let $S_{n}$ be the symmetric group on $n$ letters $1,2, \ldots, n$. For $\sigma \in S_{n}$ with $\sigma(i)=r_{i}, 1 \leqslant i \leqslant n$, we denote by

$$
\left[a_{1}, \ldots, a_{n} \mid r_{1}, \ldots, r_{n}\right] \text { or } \quad\left[a_{1}, \ldots, a_{n} \mid \sigma\right]
$$

the $n \times n$ monomial matrix with non-zero entries $a_{i}$ in the $\left(i, r_{i}\right)$-positions. For $p \mid m$ (read " $p$ divides $m$ ") in $\mathbb{N}$, we set

$$
G(m, p, n)=\left\{\left[a_{1}, \ldots, a_{n} \mid \sigma\right] \in \mathrm{GL}(\mathbb{C}, n) \mid a_{i}^{m}=1 ;\left(\prod_{j} a_{j}\right)^{m / p}=1 ; \sigma \in S_{n}\right\},
$$

where $\operatorname{GL}(\mathbb{C}, n)$ is the group of all $n \times n$ invertible matrices over the complex number field $\mathbb{C}$. $G(m, p, n)$ is an imprimitive reflection group acting on $\mathbb{C}^{n}$, which is Coxeter only when either $m \leqslant 2$ or $(m, p, n)=(m, m, 2)$. We have $G(m, p, n)=$ $G(1,1, n) \ltimes A(m, p, n)$, where $A(m, p, n)$ consists of all the diagonal matrices of $G(m, p, n)$, and $G(1,1, n) \cong S_{n}$.

1.3. We have $G(m, m, n) \subseteq G(m, 1, n)$ in general. For $q \mid m$ in $\mathbb{N}$, define a map

$$
\begin{aligned}
\phi_{m, q}^{n}: \quad G(m, 1, n) & \rightarrow G(q, 1, n), \\
\left(a_{i j}\right) & \mapsto \quad\left(a_{i j}^{m / q}\right) .
\end{aligned}
$$

Then we have $\phi_{l, q}^{n}=\phi_{m, q}^{n} \phi_{l, m}^{n}$ if $q \mid m$ and $m \mid l$.

1.4. Let $x$ be an indeterminate. Define

$$
\begin{aligned}
G(\infty, 1, n) & =\left\{\left[x^{k_{1}}, \ldots, x^{k_{n}} \mid \sigma\right] \mid k_{i} \in \mathbb{Z} ; \sigma \in S_{n}\right\} \\
G(\infty, \infty, n) & =\left\{\left[x^{k_{1}}, \ldots, x^{k_{n}} \mid \sigma\right] \in G(\infty, 1, n) \mid \sum_{j} k_{j}=0\right\} .
\end{aligned}
$$

Then $G(\infty, 1, n)$ and $G(\infty, \infty, n)$ are two matrix groups. In particular, $G(\infty, \infty, n)$ is a normal subgroup of $G(\infty, 1, n)$ with the quotient $G(\infty, 1, n) / G(\infty, \infty, n) \cong$ $(\mathbb{Z},+)$.

1.5. $x=e^{2 \pi i / m}$ specialization gives a surjection

$$
\phi_{m}^{n}: G(\infty, 1, n) \rightarrow G(m, 1, n) .
$$

By restriction, we also get surjections

$$
\begin{aligned}
\phi_{m}^{n}: G(\infty, \infty, n) & \rightarrow G(m, m, n), \\
\phi_{m, q}^{n}: G(m, m, n) & \rightarrow G(q, q, n) \text { for } q \mid m \text { in } \mathbb{N} .
\end{aligned}
$$

We have $\phi_{m}^{n}=\phi_{l, m}^{n} \phi_{l}^{n}$ on both $G(\infty, 1, n)$ and $G(\infty, \infty, n)$ for $m \mid l$. So $G(\infty, 1, n)$ (resp. $G(\infty, \infty, n))$ can be regarded as a generic version of the $G(m, 1, n)$ 's (resp. $G(m, m, n)$ 's). 
1.6. We have two inverse systems:

$$
\begin{aligned}
& \left(\{G(m, 1, n)\}_{m \in \mathbb{N}},\left\{\phi_{l, m}^{n}\right\}_{m \mid l \text { in } \mathbb{N}}\right), \\
& \left(\{G(m, m, n)\}_{m \in \mathbb{N}},\left\{\phi_{l, m}^{n}\right\}_{m \mid l \text { in } \mathbb{N}}\right) .
\end{aligned}
$$

Let $\hat{\mathbb{Z}}=\prod_{p \text { prime }}\left(\hat{\mathbb{Z}}_{p},+\right)$ be the direct product of the additive group $\left(\hat{\mathbb{Z}}_{p},+\right)$ of the completion of the $p$-adic integer ring $\mathbb{Z}_{p}$, whose composition is written multiplicatively. Let $U_{m}$ be the multiplicative group generated by the $m$ th $\operatorname{root} \zeta_{m}=e^{2 \pi i / m}$ of unity for $m \in \mathbb{N}$. Define a map $\psi_{m}: \hat{\mathbb{Z}} \longrightarrow U_{m}$ as follows. Let $m=p_{1}^{k_{1}} p_{2}^{k_{2}} \cdots p_{r}^{k_{r}}$ be the factorization into the product of distinct prime powers $p_{i}^{k_{i}}$. Then for any $a=\prod_{p} a_{p} \in \hat{\mathbb{Z}}$ with $a_{p} \in \hat{\mathbb{Z}}_{p}$, define $\psi_{m}(a)=\zeta_{m}^{k}$, where the integer $k$ is uniquely determined by the conditions $0 \leqslant k<m$ and $k \equiv a_{p_{i}}\left(\bmod p_{i}^{k_{i}}\right)$ for $1 \leqslant i \leqslant r$ according to the Chinese remainder theorem. Let

$$
\begin{gathered}
G(\hat{\mathbb{Z}}, 1, n)=\left\{\left[a_{1}, \ldots, a_{n} \mid \sigma\right] \mid a_{i} \in \hat{\mathbb{Z}} ; \sigma \in S_{n}\right\}, \\
G(\hat{\mathbb{Z}}, \hat{\mathbb{Z}}, n)=\left\{\left[a_{1}, \ldots, a_{n} \mid \sigma\right] \mid a_{i} \in \hat{\mathbb{Z}} ; \prod_{i} a_{i}=1 ; \sigma \in S_{n}\right\} .
\end{gathered}
$$

Define a map $\psi_{m}^{n}: G(\hat{\mathbb{Z}}, 1, n) \longrightarrow G(m, 1, n)($ resp. $G(\hat{\mathbb{Z}}, \hat{\mathbb{Z}}, n) \longrightarrow G(m, m, n))$ by sending $\left(a_{i j}\right)$ to $\left(\psi_{m}\left(a_{i j}\right)\right)$. One can easily check the equation $\psi_{m}^{n}=\phi_{l, m}^{n} \psi_{l}^{n}$ for any $m \mid l$ in $\mathbb{N}$. Then the inverse limit of the system (1.6.1) is $\left(G(\hat{\mathbb{Z}}, 1, n),\left\{\psi_{m}^{n}\right\}_{m \in \mathbb{N}}\right)$. Also, the inverse limit of the system $(1.6 .2)$ is $\left(G(\hat{\mathbb{Z}}, \hat{\mathbb{Z}}, n),\left\{\psi_{m}^{n}\right\}_{m \in \mathbb{N}}\right)$. The group $G(\infty, 1, n)($ resp. $G(\infty, \infty, n))$ can be embedded into $G(\hat{\mathbb{Z}}, 1, n)(\operatorname{resp} . G(\hat{\mathbb{Z}}, \hat{\mathbb{Z}}, n))$ by the specialization $x=\mathbf{e}$ (the element of $\hat{\mathbb{Z}}$ whose component in $\hat{\mathbb{Z}}_{p}$ is 1 for any prime $p$ ).

In the subsequent sections, we shall concentrate only on the groups $G(m, m, n)$, $m \in \mathbb{N}$, and their generic version $G(\infty, \infty, n)$.

\section{The ISOMORPHisM BetWeEN $G(\infty, \infty, n)$ AND $\widetilde{A}_{n-1}$}

In this section, we first show the group isomorphism $G(\infty, \infty, n) \cong \widetilde{A}_{n-1}$. Then we deduce a formula for the length function of $G(\infty, \infty, n)$ as a Coxeter group, which will be used in 3.5 to formulate a length function of the reflection group $G(m, m, n)$. Finally we show that $G(\infty, \infty, n)$ can be regarded naturally as a "Weyl group" for a certain algebraic group.

2.1. The affine Weyl group $\widetilde{A}_{n-1}, n>1$, has the permutation form (see [8, Sect. 4.1])

$$
\left\{w: \mathbb{Z} \longrightarrow \mathbb{Z} \mid(i+n) w=(i) w+n, \forall i \in \mathbb{Z} ; \sum_{i=1}^{n}(i) w=\sum_{i=1}^{n} i\right\} .
$$

We have $\widetilde{A}_{n-1} \cong W_{0} \ltimes N$ with $N=\left\{w \in \widetilde{A}_{n-1} \mid(i) w \equiv i(\bmod n), \forall i \in \mathbb{Z}\right\}$ and $W_{0}=\left\{w \in \widetilde{A}_{n-1} \mid([1, n]) w=[1, n]\right\} \cong S_{n}$, where $[a, b]:=\{a, a+1, \ldots, b\}$ for $a \leqslant b$ in $\mathbb{Z}$. 
The group $\widetilde{A}_{n-1}$ is generated by $S=\left\{t_{0}, t_{1}, \ldots, t_{n-1}\right\}$, where

$$
(i) t_{k}= \begin{cases}i, & \text { if } i \neq k, k+1(\bmod n) \\ i+1, & \text { if } i \equiv k(\bmod n) \\ i-1, & \text { if } i \equiv k+1(\bmod n)\end{cases}
$$

2.2. To each $w \in \widetilde{A}_{n-1}$, we associate an element

$$
\eta(w)=\left[x^{g_{1}}, x^{g_{2}}, \ldots, x^{g_{n}} \mid r_{1}, r_{2}, \ldots, r_{n}\right]
$$

of $G(\infty, 1, n)$ if $(i) w=g_{i} n+r_{i}$ with $g_{i}, r_{i} \in \mathbb{Z}$ and $r_{i} \in[1, n]$ for $i \in[1, n]$. By (1.4.2) and (2.1.1), we have $\eta(w) \in G(\infty, \infty, n)$. Thus $\eta$ is a map from $\widetilde{A}_{n-1}$ to $G(\infty, \infty, n)$.

Now we are given an element $X=\left[x^{h_{1}}, x^{h_{2}}, \ldots, x^{h_{n}} \mid u_{1}, u_{2}, \ldots, u_{n}\right]$ of $G(\infty, \infty, n)$. We associate to $X$ a permutation $\kappa(X)$ on $\mathbb{Z}$ as follows. Write $m=q n+r$ with $q \in \mathbb{Z}$ and $r \in[1, n]$ for any $m \in \mathbb{Z}$. We set

$$
(m) \kappa(X)=\left(q+h_{r}\right) n+u_{r}
$$

It is easily seen that $\kappa(X)$ is an element of $\widetilde{A}_{n-1}$. Moreover, we see that the maps $\eta$ and $\kappa$ are inverse to each other.

Theorem 2.3. $\eta$ is a group isomorphism from $\widetilde{A}_{n-1}$ to $G(\infty, \infty, n)$.

Proof. We have shown that $\eta$ is bijective. It remains to show that $\eta$ preserves the group multiplication. That is, to show $\eta(w) \eta(y)=\eta(w y)$ for any $w, y \in \widetilde{A}_{n-1}$. For $i, j \in[1, n]$, write $(i) w=g_{i} n+r_{i}$ and $(j) y=h_{j} n+u_{j}$ with $g_{i}, h_{j} \in \mathbb{Z}$ and $r_{i}, u_{j} \in[1, n]$. Then $(i)(w y)=\left(g_{i}+h_{r_{i}}\right) n+u_{r_{i}}$. This implies that the $(i, m)$-entry of the matrix $\eta(w y)$ is given by

$$
\eta(w y)_{i m}= \begin{cases}0, & \text { if } m \neq u_{r_{i}} \\ x^{g_{i}+h_{r_{i}}}, & \text { if } m=u_{r_{i}} .\end{cases}
$$

On the other hand, we have

$$
\begin{gathered}
\eta(w)_{i m}= \begin{cases}0, & \text { if } m \neq r_{i}, \\
x^{g_{i}}, & \text { if } m=r_{i} .\end{cases} \\
\eta(y)_{j l}= \begin{cases}0, & \text { if } l \neq u_{j}, \\
x^{h_{j}}, & \text { if } l=u_{j} .\end{cases}
\end{gathered}
$$

This implies

$$
(\eta(w) \eta(y))_{i p}= \begin{cases}0, & \text { if } p \neq u_{r_{i}} \\ x^{g_{i}+h_{r_{i}},}, & \text { if } p=u_{r_{i}}\end{cases}
$$

So we get $\eta(w) \eta(y)=\eta(w y)$, as required.

By Theorem 2.3 , we can identify $\widetilde{A}_{n-1}$ with $G(\infty, \infty, n)$. Call $\eta(w)$ the matrix form of $w \in \widetilde{A}_{n-1}$. 
2.4. We have

$$
\eta\left(t_{k}\right)=[1, \ldots, 1 \mid(k, k+1)] \quad \text { for } k \in[1, n-1],
$$

and

$$
\eta\left(t_{0}\right)=\left[x^{-1}, 1, \ldots, 1, x \mid(1, n)\right],
$$

where the notation $(i, j)$ stands for the transposition of $i$ and $j$. Moreover, the image $\eta(N)$ consists of all the diagonal matrices in $G(\infty, \infty, n)$, and $\eta\left(W_{0}\right)=G(1,1, n)$.

The length function $\ell$ of the Coxeter system $\left(\widetilde{A}_{n-1}, S\right)$ can be formulated in terms of permutation forms and of matrix forms respectively as follows.

Proposition 2.5. (1) $\ell(w)=\sum_{1 \leqslant i<j \leqslant n}\left|\left[\frac{(j) w-(i) w}{n}\right]\right|$ for $w \in \widetilde{A}_{n-1}$.

(2) For $X=\left[x^{k_{1}}, \ldots, x^{k_{n}} \mid r_{1}, \ldots, r_{n}\right] \in G(\infty, \infty, n)$, we have

$$
\ell(X)=\sum_{\substack{1 \leqslant i<j \leqslant n \\ r_{i}<r_{j}}}\left|k_{j}-k_{i}\right|+\sum_{\substack{1 \leqslant i<j \leqslant n \\ r_{i}>r_{j}}}\left|k_{j}-k_{i}-1\right|,
$$

where $[c]$ denotes the largest integer not greater than $c$, and $|c|$ is the absolute value of $c$ for any rational number $c$.

Proof. Formula (1) is given in [8, Lemma 4.2.2]. Then (2) is a consequence of (1) by applying the map $\eta$.

2.6. The group $G(\infty, \infty, n)$ can be regarded naturally as a "Weyl group" of some algebraic group $G(A)$. Let $\mathbf{k}$ be the algebraic closure of a prime field $\mathbb{F}_{p}$ of $p>0$ elements. Let $A=\mathbf{k}\left[x, x^{-1}\right], A^{+}=\mathbf{k}[x]$ and $A^{-}=\mathbf{k}\left[x^{-1}\right]$, with $x$ an indeterminate over k. Let $G=\mathrm{SL}(\mathbf{k}, n)$ be the special linear group of rank $n$ over k. For any kalgebra $D$, denote by $G(D)$ the group $\operatorname{SL}(D, n)$. Let $B, B^{*}$ be the Borel subgroups of $G$ consisting of all the upper, resp., lower triangular matrices in $G$. Then the maximal torus $T=B \cap B^{*}$ of $G$ consists of all the diagonal matrices. Define two homomorphisms $\pi^{+}: G\left(A^{+}\right) \longrightarrow G$ and $\pi^{-}: G\left(A^{-}\right) \longrightarrow G$ by specializing $x$ and $x^{-1}$ to zero respectively. Then $\widetilde{B}=\left(\pi^{+}\right)^{-1}(B)$ and $\widetilde{B^{*}}=\left(\pi^{-}\right)^{-1}\left(B^{*}\right)$ are two opposite Borel subgroups of $G(A)$ with $\widetilde{B} \cap \widetilde{B^{*}}=T$. The normalizer $N(A)$ of $T$ consists of all the monomial matrices in $G(A)$. We have $G(\infty, \infty, n) \cong N(A) / T$.

It is an open question to ask if the group $G(m, m, n)$ could also be a "Weyl group" of some algebraic group for any $m, n \in \mathbb{N}$ with $m>2$.

\section{Presentations By Generators and Relations}

In this section, we give two presentations for each of the groups $G(m, m, n)$ and $G(\infty, \infty, n)$ : one is standard, and the other is new. We point out some advantages for the new presentations of these groups at the end of the section.

3.1. The group $G(m, m, n)$ is generated by the set $S^{\prime}(m)=\left\{t_{1}, \ldots, t_{n-1}, s^{\prime}\right\}$ with $t_{k}=[1, \ldots, 1 \mid(k, k+1)]$ and $s^{\prime}=\left[e^{-2 \pi i / m}, e^{2 \pi i / m}, 1, \ldots, 1 \mid(1,2)\right] . G(m, m, n)$ can be presented abstractly by the generator set $S^{\prime}(m)$ and the following relations (see [2]):

(a) $t_{i}^{2}=e$ for $i \in[1, n-1]$, where $e$ is the identity element of the group.

(b) $t_{i} t_{i+1} t_{i}=t_{i+1} t_{i} t_{i+1}$ for $i \in[1, n-2]$;

(c) $t_{i} t_{j}=t_{j} t_{i}$ for $|i-j|>1$;

(d) $s^{\prime 2}=e$;

(e) $s^{\prime} t_{2} s^{\prime}=t_{2} s^{\prime} t_{2}$

(f) $s^{\prime} t_{i}=t_{i} s^{\prime}$ for $i \in[3, n-1]$. 
(g) $t_{1} s^{\prime} t_{2} t_{1} s^{\prime} t_{2}=t_{2} t_{1} s^{\prime} t_{2} t_{1} s^{\prime}$

(h) $s^{\prime} t_{1} s^{\prime} \cdots=t_{1} s^{\prime} t_{1} \cdots$ ( $m$ factors in both sides).

This presentation can be described by the graph in Fig.1 (see [2]).

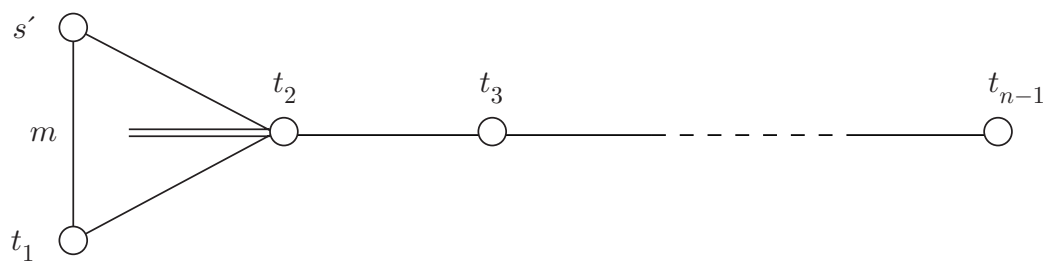

FIGURE 1.

Let $t_{0}=t_{n-1} t_{n-2} \cdots t_{2} s^{\prime} t_{2} \cdots t_{n-2} t_{n-1}$. Then

$$
t_{0}=\left[e^{-2 \pi i / m}, 1, \ldots, 1, e^{2 \pi i / m} \mid(1, n)\right] .
$$

Let $S(m)=\left\{t_{0}, t_{1}, \ldots, t_{n-1}\right\}$. For $1 \leqslant i<j<n$, let

$$
s_{i j}=t_{j} t_{j-1} \cdots t_{i+1} t_{i} t_{i+1} \cdots t_{j-1} t_{j} .
$$

Lemma 3.2. The group $G(m, m, n)$ is generated by the set $S(m)$. Moreover, we have

(i) $t_{0}^{2}=e$;

(ii) $t_{0} t_{1} t_{0}=t_{1} t_{0} t_{1}$;

(iii) $t_{0} t_{n-1} t_{0}=t_{n-1} t_{0} t_{n-1}$;

(iv) $t_{0} t_{i}=t_{i} t_{0}$ for $1<i<n-1$;

(v) $t_{0} s_{1, n-1} t_{0} \cdots=s_{1, n-1} t_{0} s_{1, n-1} \cdots \quad$ ( $m$ factors in both sides);

(vi) $t_{i} t_{i+1} \cdots t_{j-1} t_{j} t_{j-1} \cdots t_{i+1} t_{i}=t_{j} t_{j-1} \cdots t_{i+1} t_{i} t_{i+1} \cdots t_{j-1} t_{j}$ for $i \neq j$ in $[0, n-1]$, where the subscripts are regarded as the congruence classes of integers modulo $n$.

Proof. The first assertion follows directly from 3.1. Now we show the others. (i) follows by 3.1 (d) and the fact that $t_{0}$ is conjugate to $s^{\prime}$. The LHS and RHS of (ii) are equal to

and

$$
t_{n-1} t_{n-2} \cdots t_{2} s^{\prime} t_{2} t_{1} t_{2} s^{\prime} t_{2} \cdots t_{n-2} t_{n-1}
$$

$$
t_{n-1} t_{n-2} \cdots t_{3} t_{1} t_{2} s^{\prime} t_{2} t_{1} t_{3} \cdots t_{n-2} t_{n-1}
$$

respectively. Thus to show (ii), it is enough to show the equation $t_{2} s^{\prime} t_{2} t_{1} t_{2} s^{\prime} t_{2}=$ $t_{1} t_{2} s^{\prime} t_{2} t_{1}$. But the latter follows from $3.1(\mathrm{~g})$. A simple computation shows that the LHS and RHS of (iii) are equal to $s^{\prime} t_{2} t_{3} \cdots t_{n-3} t_{n-2} t_{n-3} \cdots t_{3} t_{2} s^{\prime}$ and $t_{n-2} t_{n-3} \cdots t_{2} s^{\prime} t_{2} \cdots t_{n-3} t_{n-2}$ respectively. They are equal, and hence (iii) follows. (iv) is a consequence of 3.1 (b), (c), (e) and (f). Since

$$
t_{0} s_{1, n-1}=t_{n-1} t_{n-2} \cdots t_{2} s^{\prime} t_{1} t_{2} \cdots t_{n-2} t_{n-1} \text {, }
$$

(v) follows from 3.1 (a), (h). Finally, (vi) is a consequence of 3.1 (b), (c), (e), (f) and (ii)-(iv).

Note that in the above proof, we only use the relations 3.1 (a)-(h), but not the concrete matrix forms of the elements. Now we give another presentation of the group $G(m, m, n)$. 
Proposition 3.3. The group $G(m, m, n)$ can also be presented abstractly by the generator set $S(m)$ and the following relations.

(i) $t_{i}^{2}=e$ for $i \in[0, n-1]$;

(ii) $t_{i} t_{i+1} t_{i}=t_{i+1} t_{i} t_{i+1}$ for $i \in[0, n-1]$;

(iii) $t_{i} t_{j}=t_{j} t_{i}$ for $i, j \in[0, n-1]$ with $j \not \equiv i \pm 1(\bmod n)$;

(iv) $t_{0} s_{1, n-1} t_{0} \cdots=s_{1, n-1} t_{0} s_{1, n-1} \cdots \quad$ (m factors in both sides);

where we stipulate $t_{n}=t_{0}$, and for the notation $s_{1, n-1}$ see (3.1.1).

Proof. By Lemma 3.2, it is enough to show that the relations 3.1 (d)-(h) can be deduced from the relations listed in the lemma, where $s^{\prime}:=t_{2} t_{3} \cdots t_{n-1} t_{0} t_{n-1} \cdots t_{3} t_{2}$. Note that the equation in Lemma 3.2 (vi) is a consequence of the relations (ii), (iii). So we can freely use this equation in the subsequent proof. The relation 3.1 (d) follows from (i) since $s^{\prime}$ is conjugate to $t_{0}$. For 3.1 (e), we have

$$
\begin{aligned}
s^{\prime} t_{2} & =t_{0} t_{n-1} t_{n-2} \cdots t_{4} t_{3} t_{2} t_{3} t_{4} \cdots t_{n-2} t_{n-1} t_{0} t_{2} \\
& =t_{0} t_{n-1} t_{n-2} \cdots t_{4} \cdot t_{2} t_{3} \cdot t_{4} \cdots t_{n-2} t_{n-1} t_{0} .
\end{aligned}
$$

Then the relation 3.1 (e) follows from the relations (i), (ii) since $s^{\prime} t_{2}$ and $t_{2} t_{3}$ are conjugate. The relation 3.1 (f) follows by (ii), (iii). Now we show the relation 3.1 $(\mathrm{g})$, which is equivalent to the equation

$$
\begin{aligned}
& t_{1} t_{2} t_{3} \cdots t_{n-2} t_{n-1} t_{0} t_{n-1} t_{n-2} \cdots t_{3} t_{1} t_{2} t_{3} \cdots t_{n-2} t_{n-1} t_{0} t_{n-1} t_{n-2} \cdots t_{3} \\
= & t_{2} t_{1} t_{2} t_{3} \cdots t_{n-2} t_{n-1} t_{0} t_{n-1} t_{n-2} \cdots t_{3} t_{1} t_{2} t_{3} \cdots t_{n-2} t_{n-1} t_{0} t_{n-1} t_{n-2} \cdots t_{3} t_{2}
\end{aligned}
$$

The RHS of (3.3.1) is

$$
\begin{aligned}
& t_{1} t_{2} t_{3} \cdots t_{n-2} t_{n-1} t_{0} t_{1} t_{0} t_{n-1} t_{n-2} \cdots t_{3} t_{2} t_{3} \cdots t_{n-2} t_{n-1} t_{0} t_{n-1} t_{n-2} \cdots t_{3} t_{2} \\
= & t_{1} t_{2} t_{3} \cdots t_{n-2} t_{n-1} t_{0} t_{1} \cdot t_{2} t_{3} \cdots t_{n-1} t_{0} t_{n-1} \cdots t_{3} t_{2} \cdot t_{n-1} t_{n-2} \cdots t_{3} t_{2} \\
= & t_{1} t_{2} t_{3} \cdots t_{n-2} t_{n-1} t_{0} t_{1} \cdot t_{2} t_{3} \cdots t_{n-1} t_{0} \cdot t_{n-2} t_{n-3} \cdots t_{2} t_{n-1} t_{n-2} \cdots t_{4} t_{3}
\end{aligned}
$$

by (i)-(iii). Then to show (3.3.1), it suffices to show that

$$
t_{n-1} t_{n-2} \cdots t_{3} t_{1} t_{2} t_{3} \cdots t_{n-2} t_{n-1} t_{0}=t_{1} t_{2} t_{3} \cdots t_{n-1} t_{0} t_{n-2} t_{n-3} \cdots t_{2} .
$$

But the latter can be transformed into

$$
t_{1} t_{n-1} t_{n-2} \cdots t_{3} t_{2} t_{3} \cdots t_{n-2} t_{n-1} t_{0}=t_{1} t_{2} t_{3} \cdots t_{n-1} t_{n-2} t_{n-3} \cdots t_{2} t_{0}
$$

or

$$
t_{n-1} t_{n-2} \cdots t_{3} t_{2} t_{3} \cdots t_{n-2} t_{n-1}=t_{2} t_{3} \cdots t_{n-2} t_{n-1} t_{n-2} \cdots t_{2} .
$$

The last equation holds by Lemma 3.2 (vi). This implies 3.1 (g). Finally, the relation 3.1 (h) follows from (iv) by the fact that

$$
s^{\prime} t_{1}=t_{2} t_{3} \cdots t_{n-2} t_{n-1} t_{0} t_{n-1} t_{n-2} \cdots t_{3} t_{2} \cdot t_{1}
$$

is conjugate to $t_{0} s_{1, n-1}$.

The presentation in Proposition 3.3 can be described by the graph in Fig.2. 


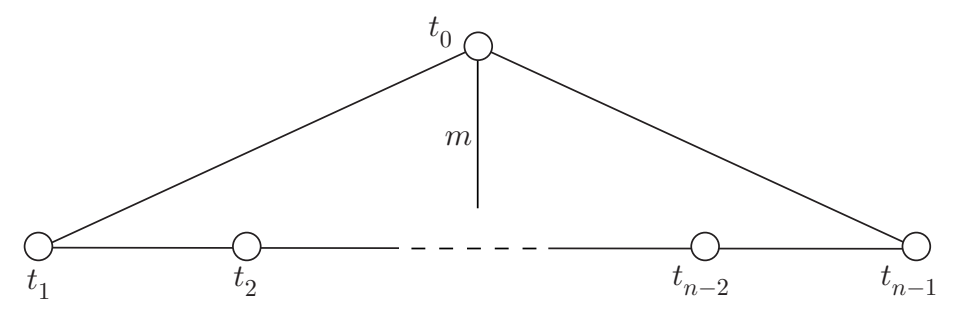

FIGURE 2.

3.4. Next we consider the presentations of the group $G(\infty, \infty, n)$. Let the monomial matrices $t_{i}, i \in[1, n-1]$, be defined as in 3.1. On the other hand, we redefine two matrices $s^{\prime}, t_{0}$ by $s^{\prime}=\left[x^{-1}, x, 1, \ldots, 1 \mid(1,2)\right]$ and $t_{0}=\left[x^{-1}, 1, \ldots, 1, x \mid(1, n)\right]$. Let $S=\left\{t_{0}, t_{1}, \ldots, t_{n-1}\right\}$ and $S^{\prime}=\left\{t_{1}, t_{2}, \ldots, t_{n-1}, s^{\prime}\right\}$. Then $G(\infty, \infty, n)$ is generated by the set $S$ or $S^{\prime}$. It can be defined abstractly by one of the following presentations.

(1) The generator set $S$ and the relations in Proposition 3.3 (i)-(iii). The corresponding graph is in Fig.3.

(2) The generator set $S^{\prime}$ and the relations 3.1 (a)-(g). The corresponding graph is in Fig.4.

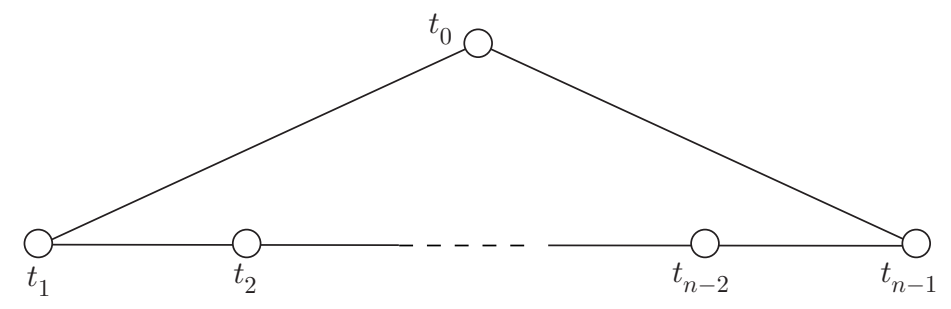

FIGURE 3.

Here presentation (1) is standard by regarding $G(\infty, \infty, n)$ as a Coxeter group (see [5], [8]), while presentation (2) is new by regarding $G(\infty, \infty, n)$ only as a reflection group. These two presentations are compatible with those of $G(m, m, n)$ respectively in the sense that the latter can be regarded as a quotient of the former modulo a certain relation. In particular, the second presentation of $G(\infty, \infty, n)$ has the advantage that a lower rank member in this family is naturally a subgroup of a higher rank member.

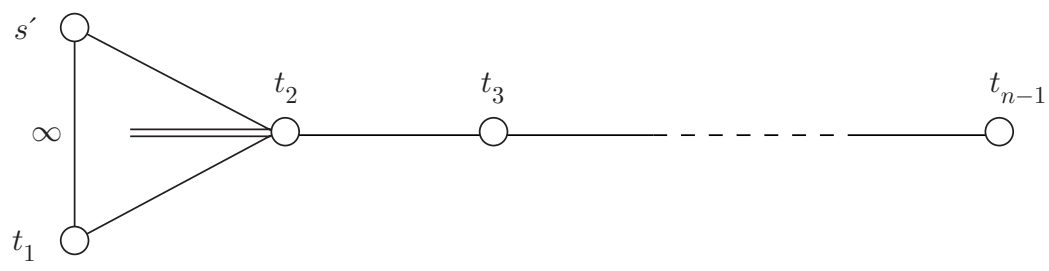

FIGURE 4. 
3.5. The presentation of $G(m, m, n)$ in Proposition 3.3 has the advantage that the length function $\ell_{m}$ on the elements of $G(m, m, n)$ with respect to the generator set $S(m)$ can be described explicitly. As usual, for $w \in G(m, m, n), \ell_{m}(w)$ is defined to be the smallest number $r$ such that $w=s_{1} s_{2} \cdots s_{r}$ with all $s_{i}$ in $S(m)$, where we stipulate $\ell_{m}(e)=0$ for the identity element $e$ of $G(m, m, n)$. We may assume $m \geqslant 2$, since a formula for the length function on $G(1,1, n)$ is already known. Express any $X \in G(m, m, n)$ in the form

$$
X=\left[\zeta_{m}^{k_{1}}, \zeta_{m}^{k_{2}}, \ldots, \zeta_{m}^{k_{n}} \mid r_{1}, r_{2}, \ldots, r_{n}\right]
$$

with $\zeta_{m}=e^{2 \pi i / m}$, where the integers $k_{j}$ are determined by $X$ up to congruence modulo $m$, and satisfy $\sum_{j=1}^{n} k_{j} \equiv 0(\bmod m)$. When $\left(k_{1}, k_{2}, \ldots, k_{n}\right)$ ranges over all the possible integer sequences satisfying (3.5.1) with the additional condition that

$$
\sum_{j=1}^{n} k_{j}=0
$$

$\ell_{m}(X)$ is the minimal possible value given by the formula (2.5.1). We can even impose one more additional condition on the range of $\left(k_{1}, k_{2}, \ldots, k_{n}\right)$ in the minimalizing process of computing $\ell_{m}(X)$. That is,

$$
k_{l}-k_{s} \leqslant m+1 \text {, }
$$

where $l, s \in[1, n]$ satisfy $k_{l}=\max \left\{k_{j} \mid j \in[1, n]\right\}$ and $k_{s}=\min \left\{k_{j} \mid j \in[1, n]\right\}$. For, suppose $k_{l}-k_{s} \geqslant m+2$. Then by replacing $k_{l}, k_{s}$ by $k_{l}-m, k_{s}+m$ in the formula (2.5.1) respectively, the resulting value will be not greater than the initial one. To see this, it is enough to check the inequality

$$
\left|k_{l}-m-k-\epsilon\right|+\left|k_{s}+m-k-\delta\right| \leqslant\left|k_{l}-k-\epsilon\right|+\left|k_{s}-k-\delta\right|
$$

for any $k_{s} \leqslant k \leqslant k_{l}$ and $\epsilon, \delta \in\{0,1,-1\}$. But this is straightforward.

Under the conditions (3.5.1)-(3.5.3), we see that each $k_{i}$ is in the interval $[-m, m]$ and has at most two possible choices in the calculation of $\ell_{m}(X)$.

3.6. Let us introduce the associated braid groups of $G(m, m, n)$ and $G(\infty, \infty, n)$. By the same argument as that for Proposition 3.3, we can show that the group presented by

(3.6.1). the generators $t_{1}, \ldots, t_{n-1}, s^{\prime}$ and the relations 3.1 (b), (c), (e)-(h). is isomorphic to that presented by

(3.6.2). the generators $t_{0}, t_{1}, \ldots, t_{n-1}$ and the relations in Proposition 3.3 (ii)-(iv). Denote both groups by $B(m, m, n)$ (up to isomorphism) and call it the associated braid group of $G(m, m, n)$. Also, the group presented by

(3.6.3). the generators $t_{0}, t_{1}, \ldots, t_{n-1}$ and the relations in Proposition 3.3 (ii)-(iii) is isomorphic to that presented by

(3.6.4). the generators $t_{1}, \ldots, t_{n-1}, s^{\prime}$ and the relations 3.1 (b), (c), (e)-(g).

Denote both groups by $B(\infty, \infty, n)$ (up to isomorphism) and call it the associated braid group of $G(\infty, \infty, n)$. 


\section{HeCKE ALGEBRAS}

To two presentations of the group $G(\infty, \infty, n)$ in 3.4 , we shall associate Hecke algebras $\mathcal{H}(G(\infty, \infty, n))$ and $\mathcal{H}^{\prime}(G(\infty, \infty, n))$ respectively. We show that they are actually isomorphic. Accordingly, we shall define two Hecke algebras associated to two presentations of the group $G(m, m, n)$ as certain quotient algebras of $\mathcal{H}(G(\infty, \infty, n))$ and $\mathcal{H}^{\prime}(G(\infty, \infty, n))$, respectively.

4.1. The Hecke algebra $\mathcal{H}(B(\infty, \infty, n))$ of the braid group $B(\infty, \infty, n)$ with respect to the presentation (3.6.3) is an associative algebra over $\mathcal{B}=\mathbb{Z}\left[q, q^{-1}\right]$ ( $q$ an indeterminate) with the unity 1 , presented by the generator set $\left\{T_{i}, T_{i}^{-1} \mid i \in[0, n-1]\right\}$ and the relations:

(1) $T_{i}^{-1} T_{i}=T_{i} T_{i}^{-1}=1$ for $i \in[0, n-1]$;

(2) $T_{i} T_{i+1} T_{i}=T_{i+1} T_{i} T_{i+1}$ for $i \in[0, n-1]$;

(3) $T_{i} T_{j}=T_{j} T_{i}$ for $i \not \equiv j \pm 1(\bmod n)$,

where we stipulate $T_{n}=T_{0}$. Then the Hecke algebra $\mathcal{H}(G(\infty, \infty, n))$ of the group $G(\infty, \infty, n)$ with respect to the presentation 3.4 (1) is an associative algebra over $\mathcal{B}$ with the unity 1 , presented by the generator set $\left\{T_{i} \mid i \in[0, n-1]\right\}$ and the relations (2)-(4), where

(4) $\left(T_{i}-q\right)\left(T_{i}+1\right)=0$ for $i \in[0, n-1]$.

Note that all the $T_{i}$ 's are invertible in $\mathcal{H}(G(\infty, \infty, n))$.

4.2. We can define another Hecke algebra $\mathcal{H}^{\prime}(B(\infty, \infty, n))$ of the group $B(\infty, \infty, n)$ with respect to the presentation (3.6.4). This is an associative algebra over $\mathcal{B}$ with the unity 1 presented by the generator set $\left\{T_{i}^{\prime}, T_{i}^{{ }^{-1}}, S^{\prime}, S^{\prime-1} \mid i \in[1, n-1]\right\}$ and the relations:

(i) $T_{i}^{\prime-1} T_{i}^{\prime}=T_{i}^{\prime} T_{i}^{\prime-1}=1$ for $i \in[1, n-1]$;

(ii) $S^{\prime} S^{\prime-1}=1$;

(iii) $T_{i}^{\prime} T_{i+1}^{\prime} T_{i}^{\prime}=T_{i+1}^{\prime} T_{i}^{\prime} T_{i+1}^{\prime}$ for $i \in[1, n-2]$;

(iv) $T_{i}^{\prime} T_{j}^{\prime}=T_{j}^{\prime} T_{i}^{\prime}$ for $|j-i|>1$;

(v) $S^{\prime} T_{2}^{\prime} S^{\prime}=T_{2}^{\prime} S^{\prime} T_{2}^{\prime}$;

(vi) $S^{\prime} T_{j}^{\prime}=T_{j}^{\prime} S^{\prime}$ for $j \in[3, n-1]$;

(vii) $T_{1}^{\prime} S^{\prime} T_{2}^{\prime} T_{1}^{\prime} S^{\prime} T_{2}^{\prime}=T_{2}^{\prime} T_{1}^{\prime} S^{\prime} T_{2}^{\prime} T_{1}^{\prime} S^{\prime}$.

Also, another Hecke algebra $\mathcal{H}^{\prime}(G(\infty, \infty, n))$ of the group $G(\infty, \infty, n)$ with respect to the presentation $3.4(2)$ is an associative algebra over $\mathcal{B}$ with the unity 1 presented by the generator set $\left\{T_{i}^{\prime}, S^{\prime} \mid i \in[1, n-1]\right\}$ and the relations (iii)-(ix), where

(viii) $\left(T_{i}^{\prime}-q\right)\left(T_{i}^{\prime}+1\right)=0$ for $i \in[1, n-1]$;

(ix) $\left(S^{\prime}-q\right)\left(S^{\prime}+1\right)=0$.

Clearly, $S^{\prime}$ and $T_{i}^{\prime}, i \in[1, n-1]$, are invertible in $\mathcal{H}^{\prime}(G(\infty, \infty, n))$.

We have the following relations among these Hecke algebras.

Proposition 4.3. (1) $\mathcal{H}^{\prime}(B(\infty, \infty, n)) \cong \mathcal{H}(B(\infty, \infty, n))$;

(2) $\mathcal{H}^{\prime}(G(\infty, \infty, n)) \cong \mathcal{H}(G(\infty, \infty, n))$.

Proof. We first show (1). Set $\phi\left(T_{i}^{\prime \pm 1}\right)=T_{i}^{ \pm 1}$ for $i \in[1, n-1]$ and $\phi\left(S^{\prime \pm 1}\right)=$ $X T_{0}^{ \pm 1} X^{-1}$, where $X=T_{2} T_{3} \cdots T_{n-1}$. We want to show that $\phi$ can be extended to an algebra homomorphism from $\mathcal{H}^{\prime}(B(\infty, \infty, n))$ to $\mathcal{H}(B(\infty, \infty, n))$. It is enough to show the following relations.

(a) $X T_{0} X^{-1} \cdot T_{2} \cdot X T_{0} X^{-1}=T_{2} \cdot X T_{0} X^{-1} \cdot T_{2}$;

(b) $T_{1} \cdot X T_{0} X^{-1} \cdot T_{2} T_{1} \cdot X T_{0} X^{-1} \cdot T_{2}=T_{2} T_{1} \cdot X T_{0} X^{-1} \cdot T_{2} T_{1} \cdot X T_{0} X^{-1}$; 
(c) $X T_{0} X^{-1} \cdot T_{j}=T_{j} \cdot X T_{0} X^{-1}$ for $j \in[3, n-1]$.

Applying the equation

$$
T_{j}^{-1} T_{j-1}^{-1} \cdots T_{i+1}^{-1} T_{i} T_{i+1} \cdots T_{j}=T_{i} T_{i+1} \cdots T_{j-1} T_{j} T_{j-1}^{-1} \cdots T_{i}^{-1}
$$

for $1 \leqslant i<j \leqslant n-1$, we see that the equation (a) is equivalent to

$$
\begin{aligned}
& T_{2} T_{3} \cdots T_{n-1} T_{0} \cdot T_{2} T_{3} \cdots T_{n-2} T_{n-1} T_{n-2}^{-1} \cdots T_{3}^{-1} T_{2}^{-1} \cdot T_{0} \\
= & T_{2}^{2} T_{3} \cdots T_{n-1} T_{0} T_{n-1}^{-1} \cdots T_{3}^{-1} \cdot T_{2} T_{3} \cdots T_{n-1} .
\end{aligned}
$$

This is further equivalent to

$$
T_{3} \cdots T_{n-1} T_{0} \cdot T_{2} T_{3} \cdots T_{n-2} T_{n-1} T_{0}=T_{2} T_{3} \cdots T_{n-1} T_{0} T_{2} T_{3} \cdots T_{n-2} T_{n-1} .
$$

But the last equation follows by applying the relations $T_{k} \cdot X T_{0}=X T_{0} \cdot T_{k-1}$ repeatedly for $k \in\{0,3,4, \ldots, n-1\}$. Next by applying the equation $T_{2} T_{1} X=$ $T_{1} X T_{1}$, we see that the equation (b) is equivalent to

$$
T_{0} X^{-1} T_{1} X T_{1} T_{0} X^{-1} T_{2} X=T_{1} T_{0} X^{-1} T_{1} X T_{1} T_{0},
$$

which is further equivalent to

$$
\begin{aligned}
& T_{0} \cdot T_{1} T_{2} \cdots T_{n-2} T_{n-1} T_{n-2}^{-1} \cdots T_{1}^{-1} \cdot T_{1} T_{0} \cdot T_{2} T_{3} \cdots T_{n-2} T_{n-1} T_{n-2}^{-1} \cdots T_{3}^{-1} T_{2}^{-1} \\
= & T_{1} T_{0} \cdot T_{1} T_{2} \cdots T_{n-2} T_{n-1} T_{n-2}^{-1} \cdots T_{2}^{-1} T_{1}^{-1} \cdot T_{1} T_{0}
\end{aligned}
$$

by repeatedly applying (4.3.1). But the last equation can be simplified into

$$
T_{n-1} T_{0} T_{n-1}=T_{0} T_{n-1} T_{0}
$$

after a certain cancelation, which is obviously true. This implies (b).

The equation (c) follows easily by the facts $X T_{j-1}=T_{j} X$ and $T_{j-1} T_{0}=T_{0} T_{j-1}$ for $j \in[3, n-1]$. So $\phi$ can be extended to an algebra homomorphism from $\mathcal{H}^{\prime}(B(\infty, \infty, n))$ to $\mathcal{H}(B(\infty, \infty, n))$; the latter is still denoted by $\phi$. Now we shall show that $\phi$ is an isomorphism. Define $\phi^{\prime}\left(T_{i}^{ \pm 1}\right)=T_{i}^{ \pm 1}$ for $i \in[1, n-1]$ and $\phi^{\prime}\left(T_{0}^{ \pm 1}\right)=Y^{-1} S^{\prime \pm 1} Y$, where $Y=T_{2}^{\prime} T_{3}^{\prime} \cdots T_{n-1}^{\prime}$. We want to show that $\phi^{\prime}$ can be extended to the inverse map of the homomorphism $\phi$. It is enough to show the following relations.

(d) $Y^{-1} S^{\prime} Y \cdot T_{j}^{\prime} \cdot Y^{-1} S^{\prime} Y=T_{j}^{\prime} \cdot Y^{-1} S^{\prime} Y \cdot T_{j}^{\prime}$ for $j=1, n-1$.

(e) $Y^{-1} S^{\prime} Y \cdot T_{k}=T_{k} \cdot Y^{-1} S^{\prime} Y$ for $k \in[2, n-2]$.

When $j=1$, the equation (d) is equivalent to

$$
T_{2}^{\prime-1} S^{\prime} T_{2}^{\prime} T_{1}^{\prime} T_{2}^{\prime-1} S^{\prime} T_{2}^{\prime}=T_{1}^{\prime} T_{2}^{\prime-1} S^{\prime} T_{2}^{\prime} T_{1}^{\prime}
$$

which follows from 4.2 (vii). When $j=n-1$, (d) is equivalent to

$$
\begin{aligned}
T_{n-1}^{\prime-1} \cdots T_{2}^{\prime-1} S^{\prime} T_{2}^{\prime} \cdots T_{n-2}^{\prime} T_{n-1}^{\prime} T_{n-2}^{\prime-1} \cdots T_{2}^{\prime-1} S^{\prime} T_{2}^{\prime} \cdots T_{n-1}^{\prime} \\
\quad=T_{n-2}^{\prime-1} \cdots T_{2}^{\prime-1} S^{\prime} T_{2}^{\prime} \cdots T_{n-2}^{\prime} T_{n-1}^{\prime 2} .
\end{aligned}
$$

The latter can be shown by repeatedly applying the relations

$$
T_{k}^{\prime-1} \cdots T_{2}^{\prime-1} S^{\prime} T_{2}^{\prime} \cdots T_{k}^{\prime}=S^{\prime} T_{2}^{\prime} \cdots T_{k-1}^{\prime} T_{k}^{\prime} T_{k-1}^{\prime-1} \cdots T_{2}^{{ }^{-1}} S^{\prime-1}
$$

for $k \in[2, n-1]$. Finally, the equation (e) follows by the facts that $Y T_{k}=T_{k+1} Y$ and $T_{k+1} S^{\prime}=S^{\prime} T_{k+1}$ for $k \in[2, n-2]$.

So (1) is shown. For $(2)$, let $\phi\left(T^{\prime}{ }_{i}\right)=T_{i}, \phi^{\prime}\left(T_{i}\right)=T_{i}^{\prime}$ for $i \in[1, n-1]$, and $\phi\left(S^{\prime}\right)=$ $X T_{0} X^{-1}, \phi^{\prime}\left(T_{0}\right)=Y^{-1} S^{\prime} Y$ with $X, Y$ as before. Then we can show $(2)$ by the same argument as that for $(1)$, and by checking the relations $\left(\phi\left(S^{\prime}\right)-q\right)\left(\phi\left(S^{\prime}\right)+1\right)=0$, 
$\left(\phi\left(T_{i}^{\prime}\right)-q\right)\left(\phi\left(T_{i}^{\prime}\right)+1\right)=0$ and $\left(\phi^{\prime}\left(T_{j}\right)-q\right)\left(\phi^{\prime}\left(T_{j}\right)+1\right)=0$ for $i \in[1, n-1]$ and $j \in[0, n-1]$

By Proposition 4.3, we can identify $\mathcal{H}(B(\infty, \infty, n))$ with $\mathcal{H}^{\prime}(B(\infty, \infty, n))$ (resp. $\mathcal{H}(G(\infty, \infty, n))$ with $\left.\mathcal{H}^{\prime}(G(\infty, \infty, n))\right)$ and call it the Hecke algebra of the group $B(\infty, \infty, n)$ (resp. $G(\infty, \infty, n))$. Clearly, the Hecke algebra of $G(\infty, \infty, n)$ is a quotient of that of $B(\infty, \infty, n)$.

4.4. The Hecke algebra $\mathcal{H}(B(m, m, n))$ (resp. $\mathcal{H}(G(m, m, n)))$ of $B(m, m, n)$ (resp. $G(m, m, n))$ also has two equivalent presentations: one is as the quotient of $\mathcal{H}(B(\infty, \infty, n))$ (resp. $\mathcal{H}(G(\infty, \infty, n)))$ modulo the relation

$$
\underbrace{T_{0} R T_{0} \cdots \cdots}_{m \text { factors }}=\underbrace{R T_{0} R \cdots \cdots}_{m \text { factors }},
$$

where $R=T_{1} T_{2} \cdots T_{n-2} T_{n-1} T_{n-2}^{-1} \cdots T_{2}^{-1} T_{1}^{-1}$. The other is as the quotient of $\mathcal{H}^{\prime}(B(m, m, n))$ (resp. $\left.\mathcal{H}^{\prime}(G(m, m, n))\right)$ modulo the relation

$$
\underbrace{T_{1}^{\prime} S^{\prime} T_{1}^{\prime} \cdots \cdots}_{m \text { factors }}=\underbrace{S^{\prime} T_{1}^{\prime} S^{\prime} \cdots \cdots}_{m \text { factors }} .
$$

\section{The Root systems of $G(\infty, \infty, n)$ And $G(m, m, n)$}

We shall give a new description for the root system $\bar{\Phi}$ of the affine Weyl group $G(\infty, \infty, n)$. The action of $G(\infty, \infty, n)$ on $\bar{\Phi}$ is natural and compatible with that of $G(m, m, n)$ on its root system $\Phi(m)$ via the pre-root system $\Phi$ and the specialization $x=e^{2 \pi i / m}$.

5.1. Let $e_{1}, \ldots, e_{n}$ be the canonical basis of the vector space $\mathbb{C}^{n}$. Let $\zeta_{m}=e^{2 \pi i / m}$ and $\delta_{i, j ; h, k}=\frac{1}{\sqrt{2}}\left(\zeta_{m}^{h} e_{i}-\zeta_{m}^{k} e_{j}\right)$. Then

$$
\Phi(m)=\left\{\delta_{i, j ; h, k} \mid i \neq j \text { in }[1, n] ; h, k \in[0, m-1]\right\}
$$

forms a root system of $G(m, m, n)$ on which $G(m, m, n)$ acts naturally (see [3]).

5.2. We shall give a new description for the root system of $G(\infty, \infty, n)$. Let $\mathcal{A}=$ $\mathbb{C}\left[x, x^{-1}\right]$ be the ring of the Laurent polynomials in an indeterminate $x$ with complex coefficients. Define an involution - in $\mathcal{A}$ by $\overline{\sum_{i} a_{i} x^{i}}=\sum_{i} \overline{a_{i}} x^{-i}$, where $\overline{a_{i}}$ is the complex conjugate of $a_{i} \in \mathbb{C}$.

Let $E$ be a free $\mathcal{A}$-module with the basis $e_{1}, \ldots, e_{n}$. Then $E$ has a complex space structure of infinite dimension which contains $\mathbb{C}^{n}$ as a subspace. Define a pairing $(\mid): E \times E \longrightarrow \mathcal{A}$ by setting $(f \mid g)=\sum_{k} f_{k} \overline{g_{k}}$ for $f=\sum_{i} f_{i} e_{i}, g=\sum_{j} g_{j} e_{j}$ in $E$. It is $\mathcal{A}$-linear in the first factor $f$ and $\mathcal{A}$-semilinear in the second factor $g$. Its restriction to $\mathbb{C}^{n}$ is a unitary inner product.

Let $\Phi$ be the set of all the elements $\gamma_{i, j ; h, k}=\frac{1}{\sqrt{2}}\left(x^{h} e_{i}-x^{k} e_{j}\right)$ in $E$ with $i \neq j$ in $[1, n]$ and $h, k \in \mathbb{Z}$. Clearly, we have $(\alpha \mid \alpha)=1$ for $\alpha \in \Phi$. Define $s_{\alpha}^{\prime}: E \longrightarrow E$ by $s_{\alpha}^{\prime}(v)=v-2(v \mid \alpha) \alpha, v \in E$, called a reflection in $E$ with respect to $\alpha$. The action of $s_{\alpha}^{\prime}$ on $E$ induces a permutation on $\Phi$.

Write $v \sim_{x} v^{\prime}$ in $E$ if $v^{\prime}=x^{k} v$ for some $k \in \mathbb{Z}$. This defines an equivalence relation in $E$. The corresponding equivalence classes of $E$ are called $x$-classes. We have $\gamma_{i, j ; h, k} \sim_{x} \gamma_{i, j ; h+1, k+1}$ for any $h, k \in \mathbb{Z}$ and $i \neq j$ in $[1, n]$. Let $\bar{E}$ be the set of $x$-classes in $E$. We see that $\Phi$ is a union of $x$-classes in $E$. Let $\bar{\Phi}$ be the set of $x$-classes of $E$ in $\Phi$. The action of $s_{\alpha}^{\prime}$ on $E$ preserves $x$-classes, and hence induces a permutation on the set $\bar{\Phi}$. 
Denote by $\bar{\alpha}$ the $x$-class of $E$ containing $\alpha$. Denote $\gamma_{i, j ; 0, k}$ by $\gamma_{i, j ; k}$. Then $\Phi_{0}=\left\{\gamma_{i, j ; k} \mid i \neq j\right.$ in $\left.[1, n] ; k \in \mathbb{Z}\right\}$ forms a representative set of $\bar{\Phi}$ in $\Phi$.

Denote $s_{i}^{\prime}=s_{\gamma_{i, i+1 ; 0}}^{\prime}, i \in[1, n-1]$, and $s_{0}^{\prime}=s_{\gamma_{n, 1 ; 1}}^{\prime}$. Then for $i \in[0, n-1]$, the matrix of $s_{i}^{\prime}$ with respect to the basis $e_{1}, \ldots, e_{n}$ is just $t_{i}$ in 3.4. By identifying $s_{i}^{\prime}$ with $t_{i}, i \in[0, n-1]$, the group $G(\infty, \infty, n)$ naturally acts on $E$ and hence on the set $\bar{\Phi}$.

We see that $\left(\overline{\gamma_{i, j ; p}}+\overline{\gamma_{k, l ; q}}\right) \cap \Phi \neq \emptyset$ if and only if either $j=k \neq i \neq l \neq j$ or $i=l \neq j \neq k \neq i$ holds. When the equivalent conditions hold, $\left(\overline{\gamma_{i, j ; p}}+\overline{\gamma_{k, l ; q}}\right) \cap \Phi$ is a single $x$-class of $E$, written $\bar{\gamma}$. More precisely, when $j=k \neq i \neq l \neq j$ (resp. $i=l \neq j \neq k \neq i$ ), we have $\bar{\gamma}=\overline{\gamma_{i, l ; p+q}}\left(\right.$ resp. $\left.\bar{\gamma}=\overline{\gamma_{k, j ; p+q}}\right)$. We regard $\bar{\gamma}$ as the sum of $\overline{\gamma_{i, j ; p}}$ and $\overline{\gamma_{k, l ; q}}$.

5.3. The following description of the affine root system of $\widetilde{A}_{n-1}$ is due to Lusztig (see [7]). Let $R=\left\{\alpha_{i j} \mid i \neq j\right.$ in $\left.[1, n]\right\}$ be the root system of $S_{n}$ satisfying $-\alpha_{i j}=\alpha_{j i}$ and $\alpha_{i t}+\alpha_{t j}=\alpha_{i j}$. Then $\Pi=\left\{\alpha_{i, i+1} \mid i \in[1, n-1]\right\}$ forms a simple root system of $R$. The sets $R^{+}=\left\{\alpha_{i j} \in R \mid i<j\right\}$ and $R^{-}=\left\{\alpha_{i j} \in R \mid i>j\right\}$ are the corresponding positive and negative root systems of $R$ respectively. Let $X=\mathbb{Z} R$ be the root lattice.

The root system of the affine Weyl group $\widetilde{A}_{n-1}=S_{n} \ltimes X$ is $\widetilde{R}=\{(\alpha, k) \mid$ $\alpha \in R ; k \in \mathbb{Z}\}$. For $p, q \in \mathbb{Z}$ and distinct $i, j, k \in[1, n]$, we have $\left(\alpha_{i k}, p+q\right)=$ $\left(\alpha_{i j}, p\right)+\left(\alpha_{j k}, q\right)$ and $-\left(\alpha_{i j}, p\right)=\left(\alpha_{j i},-p\right)$. The set

$$
\widetilde{\Pi}=\left\{\left(\alpha_{i, i+1}, 0\right) \mid i \in[1, n-1]\right\} \cup\left\{\left(\alpha_{n, 1}, 1\right)\right\}
$$

is a simple root system of $\widetilde{R}$. The corresponding positive and negative root systems of $\widetilde{R}$ are $\widetilde{R}^{+}=\{(\alpha, k) \mid \alpha \in R, k>0\} \cup\left\{(\alpha, 0) \mid \alpha \in R^{+}\right\}$and $\widetilde{R}^{-}=\{(\alpha, k) \mid \alpha \in$ $R, k<0\} \cup\left\{(\alpha, 0) \mid \alpha \in R^{-}\right\}$, respectively. Denote $\lambda \in X$ by $T_{\lambda}$ as an element of $\widetilde{A}_{n-1}$. An element $w T_{\lambda} \in \widetilde{A}_{n-1}$ acts on $\widetilde{R}$ by sending $(\alpha, k)$ to $\left(w(\alpha), k-\left\langle\lambda, \alpha^{\vee}\right\rangle\right)$, where $\langle$,$\rangle is the inner product of the euclidean space spanned by R$. Denote $\alpha_{i, j ; k}=\left(\alpha_{i j}, k\right)$ for $i \neq j$ in $[1, n]$ and $k \in \mathbb{Z}$. The reflection with respect to $\alpha_{i, j ; h}$ is $s_{\alpha_{i, j ; h}}=s_{\alpha_{i j}} T_{h \alpha_{i j}}$. Let $s_{i}=s_{\alpha_{i, i+1 ; 0}}, i \in[1, n-1]$, and $s_{0}=s_{\alpha_{n, 1 ; 1}}$. Then $S=\left\{s_{i} \mid i \in[0, n-1]\right\}$ is a distinguished generator set of $\widetilde{A}_{n-1}$ which satisfies the relations in Proposition 3.3 (i)-(iii) with $s_{i}$ in the place of $t_{i}$.

5.4. Define $\psi: \widetilde{R} \longrightarrow \Phi_{0}$ by $\psi\left(\alpha_{i, j ; k}\right)=\gamma_{i, j ; k}$ for $\alpha_{i, j ; k} \in \widetilde{R}$. This is a bijection, which induces a natural bijection: $\widetilde{R} \longrightarrow \bar{\Phi}$ (still denoted by $\psi$ ). Clearly, $\psi$ respects the additions whenever it is applicable. Moreover, under the identification of $G(\infty, \infty, n)$ with $\widetilde{A}_{n-1}$, we can denote both $s_{i} \in \widetilde{A}_{n-1}$ and $s_{i}^{\prime} \in G(\infty, \infty, n)$ by $t_{i}$ for $i \in[0, n-1]$. Then we have

$$
\psi\left(t_{i}(\alpha)\right)=t_{i}(\psi(\alpha)) \text { for } \alpha \in \widetilde{R}, i \in[0, n-1]
$$

So the map $\psi$ also respects the group actions. We call $\bar{\Phi}$ the root system of $G(\infty, \infty, n)$.

5.5. Under the specialization $x=e^{2 \pi i / m}$, the $\mathcal{A}$-module $E$ becomes the complex vector space $\mathbb{C}^{n}$, the group $G(\infty, \infty, n)$ becomes $G(m, m, n)$, and the set $\Phi$ becomes $\Phi(m)$. Also, the set $\left\{\gamma_{i, j ; k} \mid j<i\right.$ in $\left.[1, n] ; k \in \mathbb{Z}\right\}$ becomes the set $\Omega_{2}$ defined in [1] whose role in $\Phi(m)$ is analogous to a positive root system of a Coxeter group. The action of $G(\infty, \infty, n)$ on $\Phi$ is compatible with that of $G(m, m, n)$ on $\Phi(m)$ under this specialization. 


\section{ACKNOWLEDGEMENT}

I would like to express my sincere gratitute to George Lusztig, who told me that the inverse limit of the system (1.6.2) is (1.6.4), and to Bob Howlett for some useful conversations concerning the imprimitive reflection groups. I am also grateful to University of Notre Dame for financial support and hospitality during my writing of this paper.

\section{REFERENCES}

1. K. Bremke and G. Malle, Root systems and length functions, Geom. Dedicata 72(1) (1998), 83-97. MR 99g:20075

2. M. Broué, G. Malle and R. Rouquier, Complex reflection groups, braid groups, Hecke algebras, J. Reine Angew. Math. 500 (1998), 127-190. MR 99m:20088

3. Arjeh M. Cohen, Finite complex reflection groups, Ann. Scient. Éc. Norm. Sup. (4) 9 (1976), 379-436. MR 54:10437

4. M. C. Hughes, Complex reflection groups, Comm. Algebra 18(2) (1990), 3999-4022. MR 92a:20056

5. J. E. Humphreys, Reflection groups and Coxeter groups, vol. 29, Cambridge Studies in Advanced Mathematics, 1992. MR 92h:20002

6. D. Kazhdan and G. Lusztig, Schubert varieties and Poincaré duality, Proc. Sympos. Pure Math., Amer. Math. Soc., Providence, R. I. 36 (1980), 185-203. MR 84g:14054

7. G. Lusztig, Affine Hecke algebras and their graded version, J. AMS 2 (1989), 599-635. MR 90e: 16049

8. J. Y. Shi, The Kazhdan-Lusztig cells in certain affine Weyl groups, Lecture Notes in Math. vol. 1179, Springer-Verlag, 1986. MR 87i:20074

9. J. Y. Shi, Conjugacy relation for the Coxeter elements in certain Coxeter groups, Adv. in Math. 161 (2001), 1-19.

Department of Mathematics, University of Notre Dame, Notre Dame, Indiana 46556

Department of Mathematics, East China Normal University, Shanghai, 200062, P.R.C.

E-mail address: jyshi@math.ecnu.edu.cn 